\title{
Santiago Ramón y Cajal, neurocientífico y pintor
}

\author{
Santiago Ramon y Cajal, neuroscientist and painter
}

Leonardo Palacios Sánchez (1), Laura Daniela Vergara Méndez (2), Juan Pablo Liévano (3), Alejandro Guerrero (3)

\begin{abstract}
RESUMEN
La vida de este extraordinario científico y pintor ha sido motivo de estudio por generaciones, pues fascinó la manera de poder unir armoniosamente sus pasiones. Santiago Felipe Ramón y Cajal, más conocido como don Santiago Ramón y Cajal (el "don" por un título de importancia de la época e "y" como una forma de darle renombre a sus apellidos), o referido por muchos simplemente como Cajal (por la sonoridad del apellido y la costumbre en España de utilizar el apellido materno). Nació en la provincia de Cataluña, primer hijo de la unión de Justo Ramón Casasús y Antonia Cajal Puentes. Su padre, médico del pueblo, vio en él un sucesor de la profesión, razón por la cual desde muy pequeño le exigió académicamente y le reprochó su fascinación por el arte. Su infancia fue marcada por múltiples sucesos que lo llevarían a tener un encanto por la naturaleza y querer plasmarla en sus dibujos. La influencia de su padre fue exitosa, ya que se convirtió en un prestigioso médico. Ansioso de conocer otros lugares y su sed de aventurero lo llevaron a viajar a Cuba para dar apoyo médico a las tropas españolas. A su regreso se encontró con múltiples oportunidades que forjaron su carrera como científico y le dieron solidez como persona. Se casó a la edad de 27 años con Silveria Fañanás, el motor de su vida y la madre de sus seis hijos. Su incansable curiosidad y su espíritu de pintor le permitieron describir y dibujar las células nerviosas de manera detallada, incluyendo su estructura magnífica y las relaciones de estas, comparándolas de manera muy acertada con la naturaleza, referidas por él mismo como "El jardín de la neurología". Siempre fue un paisajista romántico, que se intrigó tanto de lo macro (con sus dibujos sobre la naturaleza y la anatomía), como de lo micro (la histología) (Figura 1).
\end{abstract}

PALABRAS CLAVE: Neurociencia, artista, doctrina neuronal, premio Nobel (DECS).

\section{SUMMARY}

The life of this extraordinary scientist and painter has been studied by multiple generations, fascinated by the way he harmoniously mixes his passions. Santiago Felipe Ramón y Cajal, better known as Don Santiago Ramón y Cajal (the "Don" as a title of the importance of the age and the "Y" as a way to give renown to their surnames) or referred by many simply as "Cajal" - by the sound of the surname). He was born in the province of Catalonia, first child of the union of Justo Ramón Casasús and Antonia Cajal. His father, a physician, saw in him a successor of the profession, which is why at very young age, he demanded of him the best academic performance and reproached him for his fascination with art. His childhood was marked by many events that would lead him to be charmed with nature and want to translate this into his drawings. The influence of his father was successful because he became a physician. Eager to know other places and with the thirst of an adventurer, he travelled to Cuba, but it was not the experience he had imagined. On his return he found many opportunities that forged his career as a scientist and gave him strength as a person. He married at the age of 27 years with Silveria Fañanás, who became the mother of his six children, and was the engine of his life. With his insatiable curiosity, his painter's spirit and with some help, he described in detail the nerve cells and portrayed in his drawings the magnificent structure and relationships thereof, successfully comparing them with nature, referring to "The Garden of Neurology". He was always a romantic landscape painter, intrigued as much by the macro (with his drawings of nature and anatomy) as the micro (histology); the latter was his greatest passion. All his work bore many fruits, which still amaze us today. Through his hard work and his refusal to placate his inner painter, he managed to mix his passions to their maximum potential, leaving behind a priceless legacy.

KEY WORDS. Neuroscience, Artist, Nobel Prize, Neuronal Doctrine (MeSH).

(1) Profesor titular de Neurología, Universidad del Rosario. Bogotá.

(2) Asistente de Investigación, Unidad de Neurociencia, Universidad del Rosario. Bogotá.

(3) Estudiante de Medicina, integrante del semillero de investigación en Neurociencia. Universidad del Rosario. Bogotá.

Recibido: 19/08/15. Aceptado: 8/10/15.

Correspondencia: Leonardo Palacios: leonardo.palacios@urosario.edu.co 


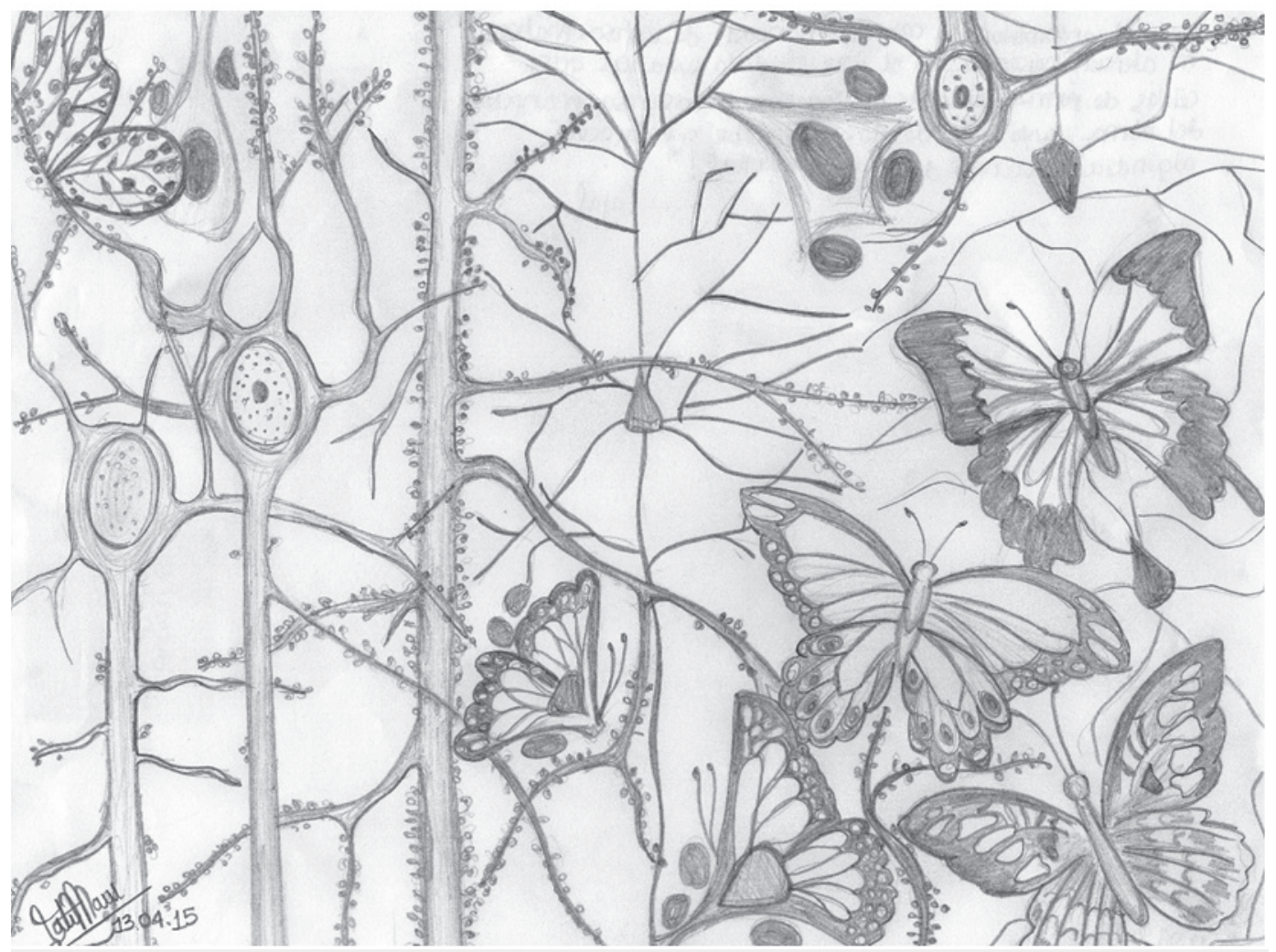

Figura 1. El jardín de la neurología. Autora: Tatiana María Luna.

Todo su trabajo dio muchos frutos, que aún nos maravillan hoy día. Con su arduo esfuerzo y negativa a aplacar su pintor interior, logró mezclar de manera potencializadora sus pasiones y dejó tras de sí un legado invaluable.

El jardín de la neurología brinda al investigador espectáculos cautivadores y emociones artísticas incomparables... y como en el vergel de la substancia gris, veía células de formas delicadas y elegantes, las mariposas del alma.

Santiago Ramón y Cajal (1852-1934)

\section{INTRODUCCIÓN}

La figura de Cajal es conocida mundialmente por sus enormes aportes en el campo de la neurociencia, sobre todo en múltiples aspectos relacionados con el tejido nervioso y específicamente con las neuronas, al punto de considerársele el padre de la "doctrina neuronal". Sus valiosos aportes le valieron múltiples distinciones en España, su país de origen, y a nivel global, siendo la más importante de ella el Premio Nobel de Medicina, que recibió en 1906, compartiéndolo con el científico italiano Camilo Golgi, "en reconocimiento a su trabajo sobre la estructura del sistema nervioso" (1).
La faceta científica e investigativa del sabio ha sido ampliamente estudiada y hay numerosas publicaciones sobre el particular. Menos conocida es la gran vocación y el enorme talento que tenía para pintar. Esta afición por la que se mostró inclinado desde muy temprana edad le permitió convertirse en el ilustrador de su propia obra. Estas breves notas pretenden ilustrar algunos aspectos sobre el arte y la ciencia en la gran figura del nobel español.

\section{Infancia y adolescencia}

Santiago Felipe Ramón y Cajal nació el primero de mayo de 1852, primogénito de Justo Ramón Casasús y Antonia Cajal Puentes (2-5), en la población de Petilla de Aragón, donde pasó sus primeros dos años, pero, según lo refiere en su obra Mi infancia y juventud, consideró su lugar de infancia Ayerbe, provincia de Huesca $(2,6,7)$. Demostró desde temprana edad talento y creatividad. Su infancia estuvo marcada por realizar un buen número de travesuras que no dejaron de ocasionar muchos problemas a sus padres, y admiración y asombro entre sus compañeros $(5,8)$. 
Inició sus estudios en una pequeña escuela de Valpalmas, en los que mostraba seguridad y habilidad (2).

Desde muy temprano mostró intereses diversos, un alma inquieta que no podía conformarse con una sola vía de estimulación. Fue así como empezó a estudiar las victorias gloriosas de España en el norte de África, que empezaron a infundir en su mente un nacionalismo que mantuvo el resto de su vida. Profundamente afectado por la caída de un rayo en el plantel educativo en el cual estudiaba y que ocasionó la muerte fulgurante de una de sus profesoras, al respecto escribió: "Por primera vez cruzó por mi espíritu profundamente conmovido, la idea del desorden y la anarquía" (8). Pocos meses después, a la edad de 8 años, pudo apreciar algo que para él constituyó una maravilla: el eclipse de sol que había sido anunciado y se produjo el 18 de julio de 1860, recorriendo gran parte de España. La experiencia acrecentó su pasión por la naturaleza; le sorprendió, además, la capacidad que tenía el hombre para predecir fenómenos naturales $(2,8)$.

A la edad de 9 años, tras su llegada a Ayerbe, villa de la Hoya de Huesca, el futuro nobel descubrió su talento en el dibujo, lo que no fue aceptado por su padre, al considerar que la pintura era una profesión de segunda clase y discrepaba con el futuro que él tenía planeado para su hijo. Don Justo Ramón Casasús soñaba en que su hijo se convirtiera, como él, en médico rural. Aun así, el rechazo de su padre solo alimentó su amor por la pintura (2). En palabras de Cajal: "Sin el misterioso atractivo del fruto prohibido las alas de la imaginación hubieran crecido, pero no hubieran llegado quizá a adquirir el desarrollo hipertrófico que alcanzaron" (6). En su obra Mi infancia y juventud dice unas palabras acerca de la opinión de su padre sobre el arte: "Prometíame que cuando fuera médico, es decir a los veintiún años de edad, asegurada mi situación económica, podría divagar cuanto quisiere por las regiones quiméricas del arte" $(6,7)$. Con notable dificultad lograba conseguir materiales para realizar sus pinturas. Se escondía en una esquina de la casa y empezaba a dibujar grandes paisajes llenos de ríos, caballos, montañas y pájaros, entre otros (2). Incluso, para aportar color a sus dibujos, Cajal recurría a su ingenio, raspando la pintura de las paredes o remojando papeles de colores solubles que le permitían extraer una gran diversidad de tonalidades (6). Para ese entonces ya era un espléndido dibujante (9). Su gran capacidad pictórica siguió viva y vigente hasta el final de su vida, dejando testimonio de ello en cuadros y en la maravillosa iconografía de sus obras, en particular disecciones anatómicas y observaciones histológicas, maravilloso ejemplo de la coexistencia de habilidades científicas y artísticas en un mismo hombre (9).

Sentía enorme atracción y admiración por la naturaleza, y tras observarla intensamente, sobre todo a los pájaros, a quienes construía jaulas de mimbre y cuidaba con gran efusión, nació su gran interés por la vida (2).

Realizó sus estudios de bachillerato en Jaca, en un colegio de padres escolapios donde estuvo un año (1862-63). Los continuó en el Instituto de Huesca, en el que se graduó como bachiller en 1869 (2). A mitad del bachillerato su padre le observó creciente fascinación por el arte y desvío de la trayectoria académica, malas notas, travesuras con la pandilla de amigos y su deseo de ser pintor $(6,10)$. Don Justo Ramón, entonces, tuvo que negociar con su hijo el permitirle tomar clases de pintura a cambio de estudiar seriamente y culminar el bachillerato. Asistió a clases de pintura con el catedrático León Abadías y, cumpliendo la promesa, terminó satisfactoriamente el bachillerato $(6,10)$. Su padre también quiso corregirle el comportamiento empleándolo como aprendiz de barbero y de zapatero remendón, con la consecuente interrupción de sus estudios por períodos largos $(2,7)$.

Cajal fue un ser visual. Además de sus grandes habilidades como pintor, a los 16 años empezó a interesarse por la fotografía, inicialmente como aficionado, luego demostró grandes habilidades en esa disciplina al tomar fotos dignas de un profesional en el área. También es llamativa la abundante cantidad de autorretratos que dejó (9).

\section{Inicio de su carrera profesional}

Su padre, médico rural, como se dijo, le inició en el estudio de la anatomía en sus períodos de vacaciones, utilizando como herramienta huesos provenientes de la fosa común del pueblo $(8,10)$. En 1869 ingresó al preparatorio de Medicina de la Universidad de Zaragoza y en 1870 inició estudios formales de Medicina. Cajal y su padre realizaron disecciones en el Hospital de Santa Engracia, en Zaragoza, durante tres años. El estudio anatómico del cuerpo comenzó a convertirse entonces para Cajal en un gran campo de interés, debido en buena parte a que encontró un punto de convergencia de sus gustos artísticos y el estudio de la medicina. Realizó dibujos de cada pieza anatómica que observaba, hecho que, por primera vez, llamó la atención de su padre, quien complacido por las asombrosas interpretaciones artísticas planteó la idea de crear un atlas analítico que mostrase lo dibujos de Cajal. Lamentablemente, el inmaduro desarrollo de las artes en Zaragoza impidió la ejecución del proyecto $(5,6)$. Obtuvo el título de licenciado en Medicina en 1873 (2).

\section{Viaje a Cuba}

En cumplimiento de una reciente disposición gubernamental sobre el año militar obligatorio ingresó al ejército y fue asignado a la provincia de Cataluña, donde permaneció 
unos meses, hasta que le llegó orden de traslado a Cuba. Esto indicaba ascenso a un grado superior: capitán (primer ayudante médico), cargo que aceptó con gusto, ansioso de aventuras en tierras desconocidas por él. Su padre intentó infructuosamente hacerlo desistir de esa idea, pues consideraba que el talento de su hijo se iba a desperdiciar. Fue así como el 17 de junio de 1874, a la edad de 22 años, llegó a La Habana $(4,8)$. Para ese entonces, a España le quedaban dos colonias en ultramar: Cuba y Puerto Rico, que ya libraban su lucha por la independencia, la cual lograrían a finales del siglo (8).

Su interés por La Habana fue creciendo, fascinándose por su arquitectura y flora tropical; además, ansiaba conocer las selvas vírgenes. Ejerció su profesión como médico en Cuba en varias poblaciones de la isla, hasta que el clima tropical, la mala alimentación y la omnipresencia de los mosquitos transmisores del paludismo disolvieron su entusiasmo al contraer malaria en junio de 1875; después de muchos trámites y en situación delicada de salud (caquexia palúdica), fue enviado de regreso a España (4).

\section{Primeros pasos como científico}

El regreso a su patria se acompañó de sentimientos de melancolía sobre el antiguo Imperio español, y de la decadencia, no solo de este, sino de la sociedad española como tal (8). De vuelta en Zaragoza y por estímulo de su padre, estudió para presentar oposiciones (presentarse a algún cargo por mérito) para la cátedra de Anatomía (4).

En 1877 aprobó los exámenes para realizar sus estudios de doctorado en Madrid, donde conoció a Aureliano Maestre de San Juan, profesor de Histología, quien desempeñó un papel definitivo en la vida del sabio, al introducirlo en el campo de la microscopia, impulsándolo a crear su propio laboratorio de histología como base para el estudio de la anatomía descriptiva (11).

En 1878 Cajal contrajo tuberculosis y se retiró a restablecer su salud en San Juan de la Peña, junto con su hermana. Deprimido y abatido por la situación, encontró consuelo y motivación en el dibujo y la fotografía. Ambos, tal y como él lo describía, lo alejaron del desagradable dolor y le aportaron una incesante tranquilidad, muy apropiada para su adecuada recuperación (12). Consiguió además el puesto de profesor auxiliar interino en la Facultad de Medicina de Zaragoza y obtuvo el título de Doctor en Medicina y Cirugía. En 1879 ganó por oposición el cargo de director de los museos anatómicos de dicha Facultad de Medicina y se casó con Silveria Fañanás García, que sería la madre de sus seis hijos $(2,4)$. Su familia fue un estímulo permanente y de gran importancia para su trabajo. Con muchas dificultades adquirió su primer microscopio y demostró una verdadera pasión por el estudio de la histología, disciplina que él bautizó "La anatomía celestial" (4, 8).

Como anatomista, particularmente interesado en el mundo de la histología, inició sus primeras observaciones y se presentó en siete oportunidades a oposiciones a fin de obtener la cátedra de Anatomía en diversas plazas. Ganó algunas y perdió otras, pero fue conquistando varias de ellas: Zaragoza, luego Valencia (1883), Barcelona (1887) y finalmente Madrid (1892) (8).

En 1889 publicó Manual de histología normal y técnica micrográfica $(2,13)$.

Su campo de acción era la histología y solo en forma ocasional trabajó en disciplinas diferentes a esta; le ocurriría, por ejemplo, en 1885, cuando Valencia era azotada por una epidemia de cólera y le asignaron como perito para dictaminar la eficacia de la vacuna del médico catalán Jaime Ferrán (1851-1929), considerando que no protegía contra la enfermedad, lo cual generó enemistad entre ellos. Posteriormente continuó su trabajo serio, permanente y muy intenso en el campo de la histología, sobre la que ya había publicado algunos artículos en revistas de circulación nacional (9).

Su primera publicación internacional fue llevada a cabo en 1886, en la Internationale Monatschrift Fur Anatomia un Physiologie, revista que dirigía el doctor Wilhelm Krause en Gotinga, Alemania (8).

En 1885, a raíz de su breve incursión en la microbiología, redactó un "Estudio sobre el microbio vírgula del cólera y las inoculaciones profilácticas", publicado por la Diputación Provincial de Zaragoza (12).

\section{Neurocientífico y artista}

En 1887, estando en la Universidad de Barcelona, inició su trabajo definitivo en torno a la histología del sistema nervioso. Su admiración por la microestructura del mismo era inmensa y permanentemente lo comparaba con elementos de la naturaleza. Refiriéndose, por ejemplo, a la dificultad para comprender la conexión entre unas células nerviosas y otras, escribió: "como rematan las raíces y las ramas de esos árboles de sustancia gris, de esa selva densa que por refinamiento de complicación carece de vacíos, de suerte que los troncos, ramas y hojas se tocan por todas partes" (8).

Posteriormente regresó a su laboratorio, en el cual hizo algunas modificaciones a la técnica de Golgi (doble coloración cromoargéntica), que aplicó para describir la anatomía histológica del sistema nervioso (retina de vertebrados, laminilla cerebelosa, lóbulo óptico de las aves, corteza cerebral y médula espinal de pequeños mamíferos; la corteza cerebral, el asta de Amón; vías ópticas, acústicas, olfatorias, y tálamos ópticos de los humanos), además de 
trabajar con embriones de pájaro (4). Lo anterior lo llevó al concepto de que el sistema nervioso está compuesto por células independientes, que se relacionan a través de sus prolongaciones (8).

En 1887 viajó a Madrid y se encontró con Luis Simarro, quien conocía la técnica que fue descrita por Camilo Golgi en 1880: la coloración cromoargéntica. Este neuropsiquiatra valenciano, que había estudiado en Francia, compartió dichos conocimientos con Cajal, quien reconoció de manera explícita su agradecimiento hacia él por su importante aporte en sus trabajos de investigación (9). Se ha postulado que el encuentro con Simarro influyó también en los intereses de Cajal sobre el campo de la psiquiatría. Cajal fundó el Comité de Investigaciones Psicológicas con el objetivo de realizar estudios de la hipnosis sugestiva, y a raíz de esto publicó en 1889 un estudio obstétrico, "Dolores de parto considerablemente atenuados por la sugestión hipnótica", primero en esta materia $(14,15)$.

En 1888, utilizando el método de Golgi, plasmó en sus dibujos por primera vez las espinas dendríticas, evidenciando su gran capacidad de interpretación de las preparaciones histológicas al dibujar diversos elementos que muchos otros científicos pasaban por alto (8).

En ese entonces el dibujo se constituía en uno de los principales medios para ilustrar las observaciones histológicas, debido a que no se poseían aún herramientas e instrumentos que permitieran plasmar de manera fidedigna la apreciación de las preparaciones. A esto se sumaba el hecho de que las técnicas de tinción, antes de la aparición del método de doble coloración cromoargéntica, no proporcionaban una imagen clara de las células nerviosas y la microglía en toda su extensión, sino que únicamente posibilitaban observar el cuerpo de ellas y partes proximales de sus prolongaciones (9).

Sin embargo Cajal, utilizando la técnica de Golgi, consiguió conjugar sus dotes artísticas con la capacidad de descifrar las preparaciones histológicas, y debido a esto obtuvo dibujos exactos y precisos de la organización y estructura neuronal en el sistema nervioso; por lo tanto, dibujar no fue una tarea sencilla, ya que el científico debió descartar aquello que consideró artefacto y dibujar lo que pensó propio del tejido, expuesto a la posibilidad de cometer desaciertos de interpretación $(13,16)$.

En la reunión anual de la Sociedad Anatómica Alemana que tuvo lugar en Berlín en octubre de 1889, Ramón y Cajal expuso su tesis y mostró sus preparaciones a científicos europeos que las observaban con curiosidad y alguna dosis de escepticismo. Se encontraba entre ellos el profesor Albert Kolliker, quien reconoció el enorme valor de lo que tenía ante sus ojos y le apoyó en forma muy importante difundiendo sus conceptos, teorías y observaciones (11).
El logro de Cajal fue inmenso; reveló la morfología de la célula nerviosa por excelencia, que fue denominada "neurona” dos años más tarde, en 1891, por el patólogo alemán Heinrich Wilhelm von Waldeyer (14) (Figura 2).

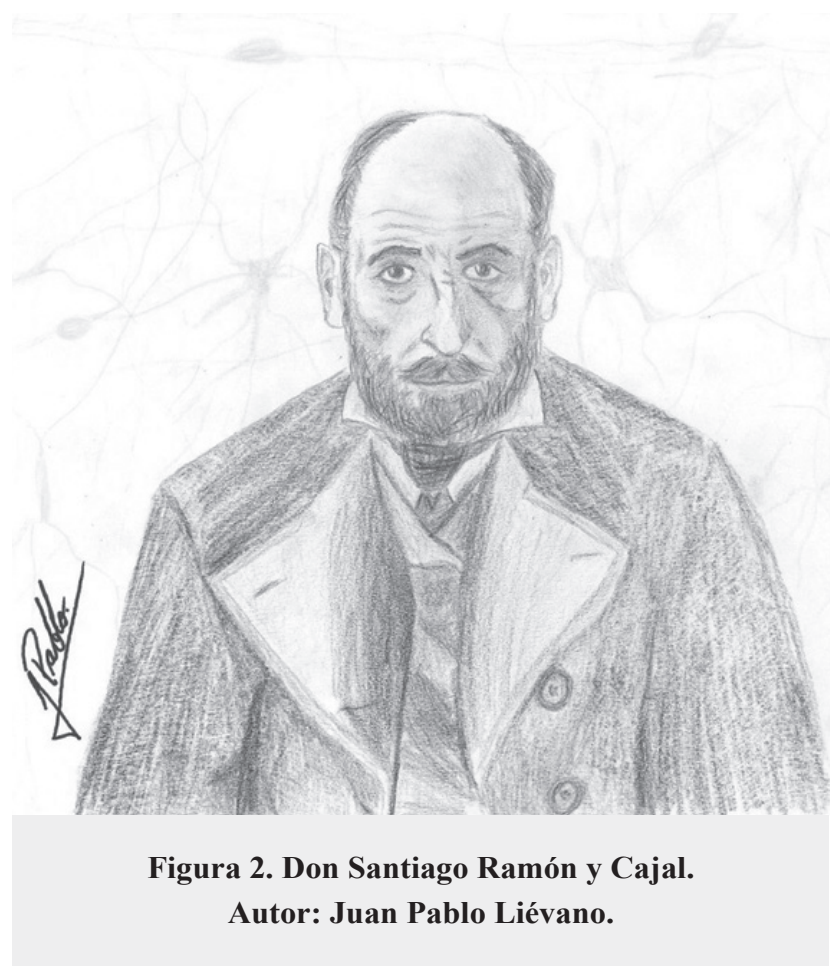

En 1892, a la edad de 40 años, retornó a Madrid para ocupar la cátedra que obtuvo por oposición. En esta época hubo dos hechos tristes de su vida: la muerte de Enriqueta, una de sus hijas, a causa de una meningitis, en 1889, y la de su hijo mayor, Santiago, quien tras enfermar gravemente murió en 1911. Cajal, aunque muy afligido, retornó rápidamente a su actividad investigativa $(2,8)$.

\section{Cajal y su técnica artística}

Haciendo uso de sus habilidades pictóricas innatas y de las adquiridas bajo la enseñanza de don León Abadías, desarrolló y configuró una técnica idónea que reflejó gran precisión, destreza y delicadeza en sus numerosos dibujos científicos acerca de la estructura del cuerpo humano y, en sí, del sistema nervioso (17).

Desempeñó su actividad artística a partir de representaciones anatómicas e histológicas, utilizándolas para divulgar sus hallazgos científicos y como herramienta para la enseñanza en sus épocas de docencia (17).

De esta manera, en la realización de sus dibujos usó dos tipos de papel: el primero era de escritura, empleado en sus obras de formato pequeño (entre $21 \mathrm{~cm}$ x 15 a $30 \mathrm{~cm}$ ); y el segundo fue papel de estraza, utilizado en sus obras de 
formato mural, que medían más de 1 metro de alto por más de $80 \mathrm{~cm}$ de ancho (17).

La mayoría de sus dibujos en formato pequeño los hacía a mano alzada: a lápiz, carboncillo, plumilla o pincel, adicionándoles posteriormente tinta china de color negro $y$, ocasionalmente, otros colores para conseguir un efecto aún más cercano a las placas histológicas que observaba. Para sus dibujos en formato mural utilizó carbón al realizar un primer bosquejo de la imagen y adicionó consecutivamente tizas de colores que servían a modo de instrumento para proveer intensidad y soporte al fondo de la imagen (17).

Para realzar las formas implementó manchas oscuras, sombrado y líneas que enfatizaban el contorno de las estructuras, sobre un fondo impregnado con aguadas (acuarelas blancas y grisáceas) que le aportaba luz y color a los dibujos. Adicionalmente, líneas pequeñas consecutivas y finos punteados con lápiz que le proporcionaban un efecto voluminoso a las imágenes. Es notable la capacidad de proveer a sus ilustraciones una sensación de profundidad, que dotaba al dibujo de una escala apropiada; demostraba la innegable vocación artística que lo acompañó durante toda su carrera científica (17).

\section{Producciones científicas y reconocimientos}

Cajal, además de publicar en varias revistas internacionales, editó una revista de publicación trimestral que él financiaba: la Revista Trimestral Micrográfica, la cual cambió de nombre e idioma varias veces a lo largo de su historia (Tabla 1). Fue invitado de honor a múltiples eventos nacionales e internacionales y empezó a recibir honores, entre ellos, el Doctorado Honoris Causa en Ciencias, de la Universidad de Cambridge, en 1894, y en Medicina de la Universidad de La Clark (Worcester, Massachusetts, Estados Unidos), en 1899, entre otras importantes distinciones (2).

Hacia el fin del siglo XIX y la entrada el XX llegó a España la época de la "Regeneración" (un movimiento intelectual y político que nació del sentimiento de decadencia por la pérdida de las colonias españolas y que buscó renovar la vida política, con base en la autenticidad de una forma objetiva, documentada y científica, al cual se incorporó Cajal) (18). Figuras como Pío Baroja, Ramón del Valle Inclán, Azorín, Menéndez y Pelayo, y otros, integraron la denominada "Regeneración" (quienes, bajo la misma idea regeneracionista, hicieron sus aportes desde un punto de vista subjetivo, más literario y artístico) (18), que marcó el renacimiento en España de una convicción en torno a la necesidad de un desarrollo cultural y científico necesario para salir de un atraso que se hizo presente desde la invasión napoleónica $(8,19)$.

En 1900 asistió en París al Congreso Internacional de Medicina, donde recibió el Premio Internacional de Moscú, galardón que se entregaba cada dos años al más importante trabajo médico o biológico en el mundo. Ese mismo año fue nombrado director del Instituto Nacional de Higiene Alfonso XII, en España (2).

En 1903 publicó en la revista Archivos Latinos de Medicina y Cirugía su trabajo "Sobre un sencillo procedimiento de impregnación de las fibras del protoplasma nervioso", que fue muy importante en torno a un fuerte debate que existía en la época sobre el tejido nervioso: un grupo apoyaba la teoría "reticularista" de Gerlach (quien consideró que el sistema nervioso estaba compuesto por redes de fibras nerviosas, sin células), entre ellos Camilo Golgi, y el otro, el de la "doctrina neuronal", liderado por Cajal, quien consideraba a la célula (que posteriormente sería denominada neurona) como componente importante del sistema nervioso. Continuó trabajando dos años más en torno al tema, hasta finalmente descubrir el botón terminal de las fibras, demostrando la validez de su doctrina $(8,17)$.

En 1905 recibió la Medalla de Oro Helmholtz, de la Academia Imperial de las Ciencias de Berlín, y en 1906 el Premio Nobel, compartido con Camilo Golgi $(1,2,10)$.

Tabla 1. Diferentes nombres que adquirió la Revista Trimestral Micrográfica

\begin{tabular}{lc}
\hline Nombre & Años \\
\hline Revista Trimestral Micrográfica & $1896-1900$ \\
\hline Trabajos del Laboratorio de Investigaciones Biológicas de la Universidad de Madrid & $1901-1906$ \\
\hline Travaux du Laboratoire de Recherches Biologiques de l’Université de Madrid & $1907-1908$ \\
\hline Trabajos del Laboratorio de Investigaciones Biológicas de la Universidad de Madrid & $1909-1923$ \\
\hline Travaux du Laboratoire de Recherches Biologiques de l’Université de Madrid & $1923-1937$ \\
\hline Trabajos del Instituto Cajal de Investigaciones Biológicas & $1940-1979$ \\
\hline
\end{tabular}

Tomado de: Gamundí (2005) (27). 
Ese mismo año tuvo el ofrecimiento, por parte de Segismundo Moret, dirigente liberal, de ocupar la cartera de Instrucción Pública con el fin de modernizar e impulsar la educación; el sabio declinó el ofrecimiento para continuar dedicado a su actividad científica (2).

Formó una pléyade de grandes histólogos, entre los que se cuentan Francisco Tello, continuador del trabajo del sabio en histología (20); Nicolás Achúcarro, quien realizó investigaciones sobre neuroglía, alcoholismo, enfermedad de Alzheimer y parálisis general (21); Gonzalo Rodríguez Lafora, neurólogo y psiquiatra, describió una forma de epilepsia mioclónica progresiva llamada posteriormente “enfermedad de Lafora" (22); Pío del Río-Hortega, médico español conocido por su descubrimiento de la microglía, cuyo epónimo es "células de Hortega", y la oligodendroglía (23); Fernando de Castro, médico español que se dedicó al estudio del cuerpo carotídeo, su histología y fisiología, gracias a lo cual recibió el Premio Nobel en 1938 (24) y Julián Sanz Ibáñez, quien fue jefe de la Sección de Oncología de la Dirección General de Sanidad y director del Instituto del Cáncer y el Instituto Cajal, del Consejo Superior de Investigaciones Científicas $(7,25)$. Cajal Apreció muchísimo a sus discípulos, se sentía estimulado por ellos y sabía que dejaría una escuela consolidada y muy productiva científicamente (13).

\section{Muerte}

A los 68 años empezó a sentirse aquejado por varios problemas de salud, pero continuó su trabajo con gran energía y dedicación (8). El sabio falleció el 17 de octubre de 1934, a los 82 años de edad (2).

La Real Academia Nacional de Medicina española organizó una exposición denominada "Cajal: Hombre y Ciencia" entre el 21 octubre de 2014 y el 20 de enero de 2015 , en la cual hubo una exposición de objetos originales pertenecientes al sabio y una lectura continuada de su obra autobiográfica Recuerdos de mi vida (26).

Agradecimientos. Al doctor Emilio Quevedo Vélez, por la revisión del artículo; al doctor Jonathan Kerr, por la revisión y corrección del resumen en inglés; a la estudiante de Medicina e integrante del Semillero de Investigación en Neurociencia, Tatiana María Luna, por el dibujo El jardín de la neurología.

\section{Conflicto de intereses}

Los autores declaran no tener conflicto de intereses.

\section{REFERENCIAS}

1. Nobelprice.org [Internet]. Suecia: Nobel media. [Actualizado en 2014; citado en septiembre 2015]. All Nobel Laureates in Physiology or Medicine. Disponible en: http://www.nobelprize.org/nobel_prizes/medicine/laureates/

2. BERBELL C. Santiago Ramón y Cajal. Rueda JM, Ed. Madrid; 1999.

3. CALVO A. Cajal, triunfar a toda costa. España; Alianza; 1999.

4. HODELÍN TABLADA R. Semblanza de don Santiago Ramón y Cajal en el 160 aniversario de su nacimiento. Rev Cubana Neurol Neurocir. 2013;3(Suppl. 1):S15-S23. [Internet] [Citado en septiembre 2015]. Disponible en: http://www.revneuro.sld. $\mathrm{cu}$

5. Serrablo.org [internet]. España: Solsona F. Justo Ramón Casasús. [Actualizado en febrero 5 de 2001; citado en septiembre de 2015]. Disponible en: http://www.serrablo.org/boletin/ s117/s117a5.html

6. RAMÓN Y CAJAL S. Mi infancia y juventud. Calpe E, Ed. Madrid; 1946.

7. MARTÍNEZ-FALERO J. Tribuna humanística: Cajal y su obra literaria. Rev Toko - Gin Pract. 2011;70(3):110-22.

8. OSORIO E. Santiago Ramón y Cajal. Boletín de la Sociedad Colombiana de Historia de la Medicina 1984 Dic;3(4):1-7.

9. ESTAÑOL B. El misterio del genio creativo de Santiago Ramón y Cajal. A los 100 años del premio nobel. Salud mental
2007;30(3):5. Disponible en: http://redalyc.org/articulo. oa?id $=58230309$

10. morfovirtual2012.sld.cu [internet]. Madrid: Segovia JAdC. Cajal, ganador del Premio Nobel. [Actualizado en 2012; citado en septiembre 2015]. Disponible en: http://www.morfovirtual2012.sld.cu/index.php/morfovirtual/index/pages/view/ nobelcajal

11. DE FELIPE J. Cajal y sus dibujos: ciencia y arte. 2005. En: Arte y neurología [Internet]. Madrid: Saned. Disponible en: http://digital.csic.es/bitstream/10261/ 12879/3/Cajal_Art. pdf

12. RAMÓN Y CAJAL JUNQUERA S. Santiago Ramón y Cajal y la patología. En: Técnica Sg, Ed. Ramón y Cajal y la ciencia española. Zaragoza, España: Ministerio de Educación y Ciencia; 2005. p. 15.

13. RAMÓN Y CAJAL S. Recuerdos de mi vida. Sánchez-Ron J, Ed. Barcelona, España: Fundación Hiberdrola; 1899.

14. LÓPEZ-MUÑOZ F, ÁLAMO C, GARCÍA-GARCÍA P, BOYA J. Relevancia histórica de la teoría neuronal un siglo después del nobel de Cajal: implicaciones psiquiátricas y psicofarmacológicas. Psiquiatría Biológica 2006;13(5):167-82.

15. LÓPEZ-MUÑOZ F, ALAMO C, MOLINA J. Los vínculos psiquiátricos en la obra y vida de Cajal. Norte de salud mental 2010;8(36):13. 
16. REGIDOR-GARCÍA J, DEFELIPE J, FRANCK G, GONZÁLEZ-RODRÍGUEZ A, GUERRA C. El cerebro, la gran cepa azul: arte y neurociencia [Internet]. Islas Canarias, España: Museo Elder de la Ciencia y la Tecnología y Gobierno de Canarias, 2010. [Actualizado en 2010; citado en septiembre 2015]. Disponible en: http://cajalbbp.cesvima.upm.es/ press/Popular\%20Science\%20III_El\%20cerebro\%20la\%20 gran $\% 20$ cepa $\% 20$ azul.pdf

17. MESEGUER J. Arte y ciencia: consideraciones artísticas sobre los dibujos histológicos de Santiago Ramón y Cajal [Tesis doctoral]. España: Universidad de Murcia; 2014. Disponible en: www.tdx.cat/bitstream/handle/10803/284726/ TJMP. pdf;jsessionid...1

18. Hispanoteca.eu [internet]. España: Fernández-López J. Regeneracionismo y krausismo. Renovación cultural de España. [Citado en septiembre 2015]. Disponible en: http://hispanoteca.eu/Literatura $\% 20$ espa $\%$ C3\%B1ola/Siglo $\%$ 20XIX/ Regeneracionismo $\% 20 \mathrm{y} \% 20 \mathrm{krausismo.htm}$

19. Jralonso.es [Internet]. España: Ramón Alonso J. Cajal, regeneracionista. [Actualizado en 2014; citado en septiembre de 2015]. Disponible en: http://jralonso.es/2014/09/22/cajalregeneracionista/

20. Ranm.es [Internet]. España: Real Academia Nacional de Medicina - 1923. Tello Muñoz, Jorge Francisco [Actualizado en 2005; citado en septiembre de 2015]. Disponible en: http://www. ranm.es/academicos/academicos-de-numero-anteriores/8461923-tello-munoz-jorge-francisco.html

21. MARTÍNEZ-AZUMENDI O. Dr. Nicolás Achucarro (1880-1918). Norte de Salud Mental 2001;4(11):4. Disponible en: http://revistanorte.es/index.php/revista/ article/ view $/ 188 / 184$
22. GONZÁLEZ-CAJAL J. Gonzalo Rodríguez Lafora: breves notas biográficas. Revista Asociación Española de Neuropsiquiatría 1998;3(27):21. Disponible en: http://documentacion. aen.es/pdf/revista-aen/1988/revista-27/09-gonzalo-rodriguez-lafora-breves-notas-biograficas.pdf.

23. CASTELLANO-LÓPEZ B. González de Mingo. Contribuciones científicas de don Pío del Río-Hortega a la neurociencia. Neurología 1995;10(7):12. Disponible en: http://histoserver. uab.es/PDFs/Publicaciones/1995_ Contribuciones $\% 20$ cient $\%$ C $3 \%$ ADficas $\% 20 \mathrm{de} \% 20$ Don $\% 20 \mathrm{P} \% \mathrm{C} 3 \%$ ADo $\% 20$ del $\% 20$ R $\%$ C3\%ADo $\% 20$ Hortega $\% 20$ en $\% 201 \mathrm{a} \% 20$ neurociencia.pdf

24. Mcnbiografías.com [internet]. España: Baratas-Díaz A., Castro Rodríguez, Fernando de (1896-1967). [Citado en septiembre 2015]. Disponible en: http://www.mcnbiografias.com/app$\mathrm{bio} / \mathrm{do} /$ show?key= castro-rodriguez-fernando-de.

25. Rac.es [internet]. España: Real Academia de Ciencias Exactas FYN. Relación de académicos desde el año 1847 hasta el 2003. [Citado en septiembre de 2015]. Disponible en: http://www. rac.es/ficheros/doc/00186.pdf.

26. Ranm.es [internet]. España: Exposición "Cajal: Hombre y Ciencia”. Real Academia Nacional de Medicina. [Actualizado en septiembre de 2014; citado en septiembre 2015]. Disponible en: http://www.ranm.es/sesiones-y-actos/proximassesiones /3739-inauguracion-de-la-exposicion-cajal-hombre-yciencia-21-de-octubre-de-2014.html

27. GAMUNDÍ A, TIMONER G, NICOLAU MC, RIAL RV, ESTEBAN S, LANGA MA. La obra de Santiago Ramón y Cajal en Revista Trimestral Micrográfica (Trabajos del Laboratorio de Investigaciones Biológicas). Rev Neurol. 40(11): 696-700. Disponible en: http://digital.csic.es/bitstream/10261/8511/1/ LangaRev.Neurol.,2005.pdf 